\title{
Efficacy of topical therapy with newly developed terbinafine and econazole formulations in the treatment of dermatophytosis in cats
}

\author{
M. Ivaskiene1, A.P. Matusevicius¹, A. Grigonis ${ }^{2}$, G. Zamokas², L. Babickaite ${ }^{2}$ \\ ${ }^{1}$ Laboratory of Experimental and Clinical Pharmacology, Department of Non-Infectious Diseases, \\ Lithuanian University of Health Sciences, Veterinary Academy, Tilzes 18, LT-47181 Kaunas, Lithuania \\ ${ }^{2}$ Dr. L Kriauceliunas Small Animal Clinics, Lithuanian University of Health Sciences, Veterinary Academy
}

\begin{abstract}
In the field of veterinary dermatology dermatophytosis is one of the most frequently occurring infectious diseases, therefore its treatment should be effective, convenient, safe and inexpensive. The aim of this study was to evaluate the efficacy of newly developed topical formulations in the treatment of cats with dermatophytosis. Evaluation of clinical efficacy and safety of terbinafine and econazole formulations administered topically twice a day was performed in 40 cats. Cats, suffering from the most widely spread Microsporum canis-induced dermatophytosis and treated with terbinafine hydrochloride $1 \%$ cream, recovered within $20.3 \pm 0.88$ days; whereas when treated with econazole nitrate $1 \%$ cream, they recovered within $28.4 \pm 1.14$ days. A positive therapeutic effect was yielded by combined treatment with local application of creams and whole coat spray with enilconazole $0.2 \%$ emulsion „Imaverol". Most cats treated with econazole cream revealed redness and irritation of the skin at the site of application. This study demonstrates that terbinafine tended to have superior clinical efficacy $(\mathrm{p}<0.001)$ in the treatment of dermatophytosis in cats compared to the azole tested.
\end{abstract}

Key words: cat, terbinafine, econazole, cream, dermatophytosis

\section{Introduction}

Dermatophytosis, also known as tinea or ringworm, is a disease caused by superficial fungal infection of the skin with a propensity to attack hair shafts and follicles. It is caused by fungi of the genera Microsporum, Trichophyton and Epidermophyton. Besides humans, it may affect rodents, dogs, cats, horses, cattle and swine. Dermatophytes are classified as zoophilic, mainly found in animals, but can be passed to humans. Anthropophilic dermatophytes are mainly found in humans and are passed to animals rarely. Geophilic dermatophytes are found mainly in soil, where they feed on decomposing hair, feathers, hooves and other sources of keratin. They infect both humans and animals. Dermatophytosis is very contagious and spreads extremely quickly among humans and animals. This disease is the most commonly occurring dermatological zoonosis. Over $90 \%$ of feline dermatophytosis cases worldwide are caused by Microsporum canis (Seebacher et al. 2008, Frymus et al. 2013). 
Today dogs and cats have become the most popular pets in the world, and suit our lifestyle perfectly. As a result, it is common for people to be in close contact with pets, who often happen to suffer from zoonosis. There is an increasing amount of publications focusing on the superficial and invasive mycosis that spread among people (Skerlev and Miklic 2010). Microsporum canis is the most common agent in Europe to cause tinea capitis in children (Seebacher et al. 2008).

Pets are frequently blamed for the transmission of dermatophytes between animals and humans. Transmission between hosts usually occurs by direct contact with a symptomatic or asymptomatic host, or direct or airborne contact with its hairs or skin scales. Infective spores in hair and dermal scales can remain viable from several months to years in the environment. Skin lesions in both humans and animals are usually characterized by inflammation that is the most severe at the edges, with erythema, scaling and occasionally blister formation. Lesions can be localized or generalized, usually pruritic in humans, not animals, with burning sensation. Dermatophytes that are acquired from animals or soil generally cause more inflammatory lesions in humans than anthropophilic dermatophytes (Ameen 2010). Although, dermatophyte infections may be self-limiting in the individual with a strong immune system and good living condition, the treatment helps to expedite the resolution of the disease and minimize the risk for infected spores to spread into the environment (Scott et al. 2001). Topical therapy is intended for animals with dermatophytosis, and may be the sole therapy for local, non-diffuse lesions (Moriello 2004).

Although mycoses are widespread, for a long time there was a limited choice of effective and non-toxic antifungal agents used for treatment (Vanden Bossche et al. 2003). Polyenes and pyrimidine derivatives were available for the treatment of mycoses; however, their limited antifungal spectrum and toxicity to mammalian cell diminished their use (Maertens 2004). The continued search for new and less toxic antifungals led to the discovery of the imidazoles, the modification of which led to the development of more potent triazoles and bistriazoles. During the last decades, a new group of allylamines has been synthesized. The antifungal action of allylamines is mediated by inhibition of ergosterol biosynthesis at a site much earlier in the pathway than the azole antifungal drugs (Matusevicius et al. 2008a,b). Allylamines are highly selective for the fungal enzyme and have a minimal effect on mammalian cholesterol synthesis, thus are more effective and less toxic to mammalian cell than azoles. Over the past years, there have been a variety of trials evaluating use of topical terbinafine addressing different pharmaceutical formulations. Terbinafine is very well tolerated in any topical pharmaceutical formulation and also has high efficacy as a cure for dermatophytosis in humans, irrespective of type of pharmaceutical formulation, treatment duration and frequency of application (Korting et al. 2007). Recently, econazole and terbinafine are widely used in antifungal preparations for human mycoses, however these agents are not licensed for use in animals. Although terbinafine is prescribed for the treatment of mycoses in humans, it is increasingly being used in veterinary patients (Sakai et al. 2011). In Lithuania, available topical formulations for use in pets are Surolan $^{\circledR}$, Malaseb ${ }^{\circledR}$ (miconazole) and Imaverol ${ }^{\circledR}$ (enilconazole), which contain antifungals of first generation imidazole, that are fungistatic, have narrow spectrum of activity and the development of resistance to these antifungals has become increasingly apparent. The use of an effective and safe antifungal therapy, which decreases the time of treatment and owner's exposure to the disease, is important in veterinary medicine.

\section{Materials and Methods}

\section{Newly developed formulations}

The Laboratory of Experimental and Clinical Pharmacology in Veterinary Academy of Lithuanian University of Health Sciences has prepared two topical formulations, E-1 and T-1, to treat pets infected with dermatophytosis. Both topical formulations in the form of cream are developed on the basis of homogeneous oil-in-water emulsion. The vehicle of formulations contains chemical substances, which are safe and commonly used in topical preparations. These substances are: salicylic acid, monoethanolamine and chloralhydrate. The formulation E-1 contains antifungal active agent belonging to imidazole group - econazole nitrate $(1 \%)$; $\mathrm{T}-1$ formulation contains agent belonging to allylamine group - terbinafine hydrochloride $(1 \%)$. Formulations have to spread easily and dry rapidly on animal's skin, leaving no detectable residue and adhering to the treated area without being tacky, having optimal $\mathrm{pH}$ and being non-irritating to the skin, as well as having no unpleasant texture or odour, but having keratolytic and moisturizing effect on skin. The aim of this study was to determine the clinical efficacy of newly designed topical formulations E-1 and $\mathrm{T}-1$. 
Animals

Scientific research was conducted according to the Act No. B1-639 of the Republic of Lithuania, dated 18/12/2008, Regarding Animal Care, Storage, Maintenance and Use („Valstybes zinios”, 22/01/2009, No 8 ). The treatment was performed by the pet's owner upon signing the consent. Effectiveness and safety of experimental creams were assessed in 40 cats (random age and gender) with $M$. canis naturally induced dermatophytosis that had from one to five clearly expressed skin lesions with a diameter of less than $5 \mathrm{~cm}$. Microsporum canis was identified in 86 damaged skin sites. The majority $(\mathrm{n}=45 ; 52.3 \%)$ of damaged skin sites was determined in the head and muzzle areas. Limb area of damaged skin amounted to $33.7 \%$ $(n=29)$, body area amounted to $11.6 \%(n=10)$ and tail area amounted to $2.3 \%(\mathrm{n}=2)$.

\section{Animal treatment}

The cats were divided into 4 experimental groups (A, B, C, D), each containing 10 animals. The cats had similar intensity of infection - extensive damage to the skin, erythema, crusting, ulceration, loss of hair. Group A was treated with cream T-1 only, group $B$ was treated with cream E-1 only, group C and D were treated with experimental creams - T-1 and E-1, respectively and additionally with enilconazole $0.2 \%$ emulsion „Imaverol” spray every 3 days until the end of clinical changes.

A pea-sized portion (average $25 \mathrm{mg}$ ) of experimental cream was used for one application. Skin lesions were treated with experimental creams two times a day, in the morning and in the evening, by rubbing it gently into the affected area before the feeding time, or Elizabethan collars were used to prevent cats from grooming. The lesions were visually examined daily throughout the experiment to determine the severity and recovery of lesion. The animal was considered to have recovered completely, when the hair in the damaged area had fully grown back, there were no signs of infection and the mycological test result was negative.

\section{Clinical evaluation}

Changes in lesion scaling, erythema, ulceration or alopecia were examined and recorded daily. To evaluate the clinical efficacy, the methodology described by Ghannoum et al. (2009) was used. The infected area was divided into four equal quadrants. Each quadrant was scored on a scale from 5 to 0 as follows: 5 - exten- sive damage to the skin, redness, crusting, ulceration, loss of hair; 4 - erythematous skin, loss of hair, scaling; 3 - slightly erythematous skin, moderate scaling, hair starts to re-grow, few bald patches; 2 - no erythema, no swelling, hair re-grows over entire lesion site, little scaling; 1 - no erythema, no scaling, hair is half length long; 0 - absence of lesion, no signs of infection, hair is fully re-grown. These scores were summed for the four sites on each animal and were used to compare the efficacy of different treatments. Treatment efficacy in percents was calculated using the following equation: Efficacy $=100-\left(T^{*} 100 / C\right)$, where $\mathrm{T}$ - the total score of treated lesion in each animal; $\mathrm{C}$ - the score of 20 for the unhealed lesion. The total score for any group denotes the average clinical score from different animals in the same group.

\section{Mycological examination}

Cats infected with Microsporum canis were identified at admission via physical examination and presence of skin lesions. Visual diagnosis was approved by performing standardised mycological test. Damaged hairs were plucked with sterile tweezers from the demarcation zone between healthy and damaged skin, scabs and dandruff were collected. The sample from each animal was divided into two parts. One part was cultivated under aerobic conditions in a thermostat at $28^{\circ} \mathrm{C}$, another part was cultivated under aerobic conditions in a thermostat at $37^{\circ} \mathrm{C}$. Samples were placed on the Sabouraud Dextrose Agar in Petri dishes, incubated for up to 7 days and examined daily. Fungal identification was based on cultural morphology and microscopic examination of hyphae, microconidia and macroconidia. Lactophenol Cotton Blue Solution was used as mounting medium and staining agent in the preparation of slides for microscopic examination of fungi. Mycological test was repeated after clinical signs of infection disappeared. MacKenzie's toothbrush technique was also used to ensure spores had not remained on the coat.

\section{Data and statistical analyses}

Statistical analysis of data was performed using SPSS statistical package (version 15, SPSS Inc., Chicago, IL). Mean total $( \pm$ SE) (SE - standard error) of treatment days was calculated for each treatment group. The groups were compared by a time-to-event analysis (survival analysis). The Log Rank (Mantel-Cox) test was used to compare the survival time. Student test Independent Samples T Test was 


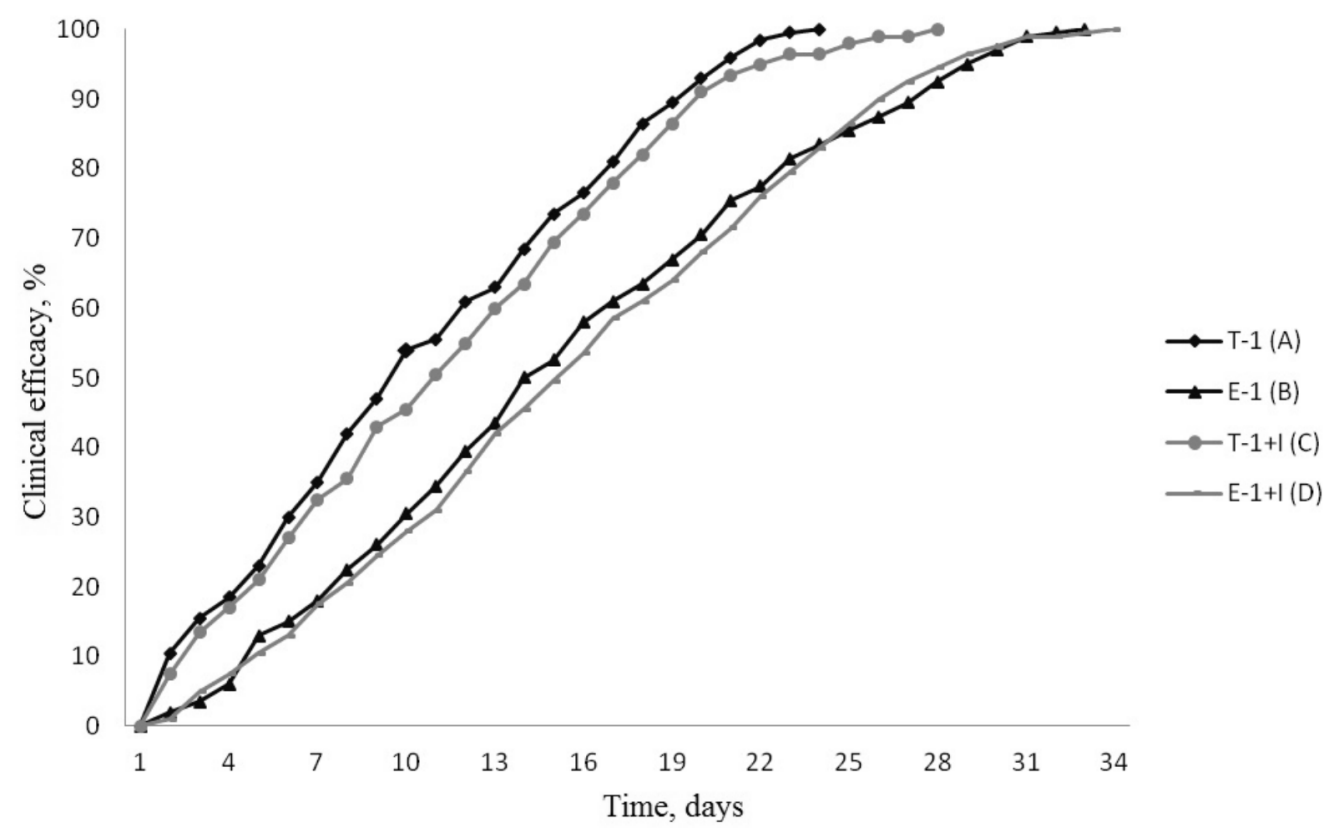

Fig. 1. Clinical efficacy of tested formulations.

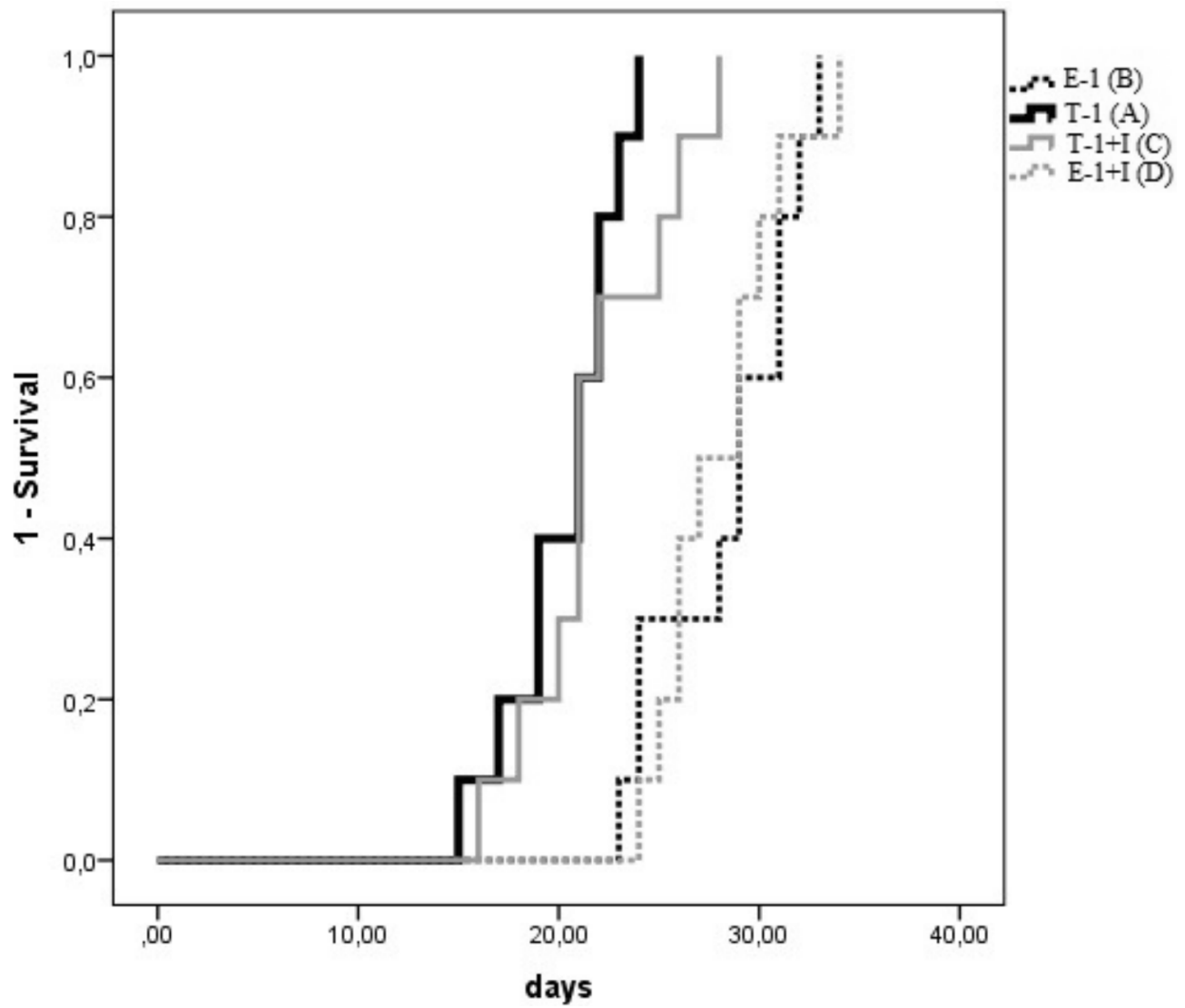

Fig. 2. Distribution of healing time according to the treatment used. 
applied to evaluate the differences between effectiveness of treatments. $\mathrm{P}$ value $<0.05$ was considered significant.

\section{Results}

Figure 1 shows comparative clinical percent efficacy of each tested formulation. First signs of recovery were seen on the second day of treatment in animals of group A and C, while group B and D showed recovery signs on the third day of the treatment. Cats, suffering from Microsporum canis-induced dermatophytosis, when treated with cream T-1 (group A), have recovered in $20.3 \pm 0.88$ days (15-24 d.); whereas, when treated with cream E-1 (group B), they have recovered in $28.4 \pm 1.14$ days (23-33 d.). During this research, two groups of cats with localized skin lesions were treated with experimental creams and ,Imaverol solution. Such a combined treatment of local application of cream T-1 and whole coat spray with „Imaverol” solution (group C) yielded positive therapeutic effect in $21.8 \pm 1.15$ days $(16-28 \mathrm{~d}$.), whereas local application of cream E-1 and whole coat spray with „Imaverol” solution (group D) yielded positive therapeutic effect in $28.1 \pm 0.97$ days (24-34 d.). The difference between mean treatment time of groups A, $\mathrm{C}$ and $\mathrm{B}, \mathrm{D}$ was statistically significant $(\mathrm{p}<0.01)$. The probability of recovery over the time is shown in Fig. 2 . Treatment with the cream T-1 reached the probability 1 faster (all treated animals recovered). This was followed by the combined treatment of cream T-1 and „Imaverol” spray, cream E-1 and ultimately by combined treatment of cream E-1 and „Imaverol” spray. Treatment with T-1 and „Imaverol”, E-1 and „Imaverol” demonstrated clinical efficacy after $6.6 \pm 1.5$ and $8.7 \pm 1.2$ applications, respectively. This study demonstrates that treatment with the cream T-1 influence the healing rate statistically significantly $(p<0.001)$ compared to the treatment with cream E-1 and combined treatment with cream E-1 and „Imaverol” solution. During observational period of 12 months, all the cats did not show disease recurrence. Figures 3-8 show the lesions on the right cheek and the right side of the neck of the 2 year old cat treated with the cream $\mathrm{T}-1$ and recovered in 15 days.

\section{Discussion}

Over the past decade, the effectiveness and tolerability of terbinafine were actively investigated in the treatment of animal dermatomycoses (Bechert et al. 2010, Sakai et al. 2011, Williams et al. 2011, Wang et al. 2012). After a number of studies in animals, scien- tists have proved that orally administered terbinafine is effective in the treatment of cats suffering from experimentally induced or naturally occurring dermatophytosis (Castanon-Olivares et al. 2001, Kotnik 2002, Kotnik and Cerne 2006, Foust et al. 2007). Recently topically applied terbinafine showed superior effectiveness in the treatment of experimental M. canis infection in guinea pigs (Ivaskiene et al. 2011).

In the present study, treated cats had a similar level of infection intensity; but the time of the disappearance of the clinical symptoms varied. It was noticed that lesions began recovering rapidly after crusts and infected hairs, both of which are the food source of dermatophytes, were removed. Presumably this happened due to everyday moisturizing effect on skin, which speeds up the cleansing of the lesion and helps to recover skin barrier properties. It was noticed that after the crust is gone and the hair had fallen off, it is easier for the pharmacological formulation to reach the stratum corneum and the microscopic fungi present within. Therefore, after such „cleansing” the lesion heals and the hairs start to grow back.

During the research most cats treated with cream E-1 revealed redness and irritation of the skin at the site of application. The animals have been starting to scratch the patch of skin, which the cream was applied to, immediately after application; however, the irritation would disappear after couple of hours. It is known that azoles used in topical formulations may cause side effects, such as skin itching, redness and burning sensation (Sheppard and Lampiris 2007).

According to printed sources, dermatophytosis is a common infectious skin disease among small animals. Dermatophytosis is highly contagious and zoonotic; therefore its treatment has to be effective, convenient, safe and inexpensive. When treating cutaneous fungal infections in pets, topical drugs are often preferred to oral drugs. The efficacy of a topical drug depends on the nature of the vehicle and the physicochemical properties of its active substance. A higher oral dose usually needs to be administered to achieve the same local concentration of a drug, which increases the risk of side effects (Ozcan et al. 2009). The preferred form for a topical administration of an active substance is cream and lotion, which are usually homogeneous oil-in-water emulsions, or „,vanishing creams" that have a continuous aqueous phase containing oily globules. Oil-in-water emulsions intensely hydrate the skin. Increased skin hydration opens the structure of the superficial layers of the skin, which in turn increases the penetration of active agents (Benson 2005). The evaporation of water provides a cooling effect on the skin (Williams 2003).

It is a common knowledge that emollients, moisturizers and keratolytic agents are central to the topi- 

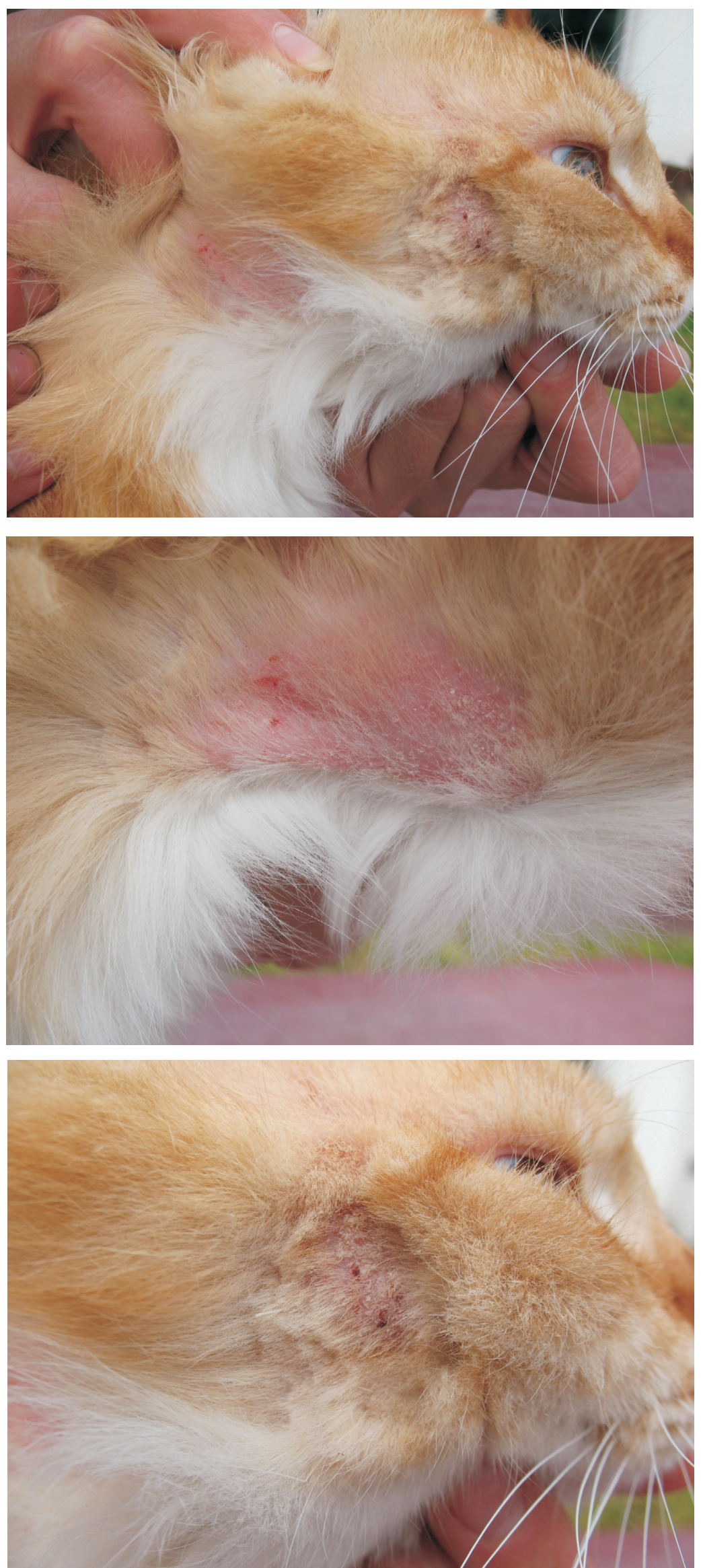

Figs. 3, 4, 5. Lesions on the first day of treatment with cream T-1. 

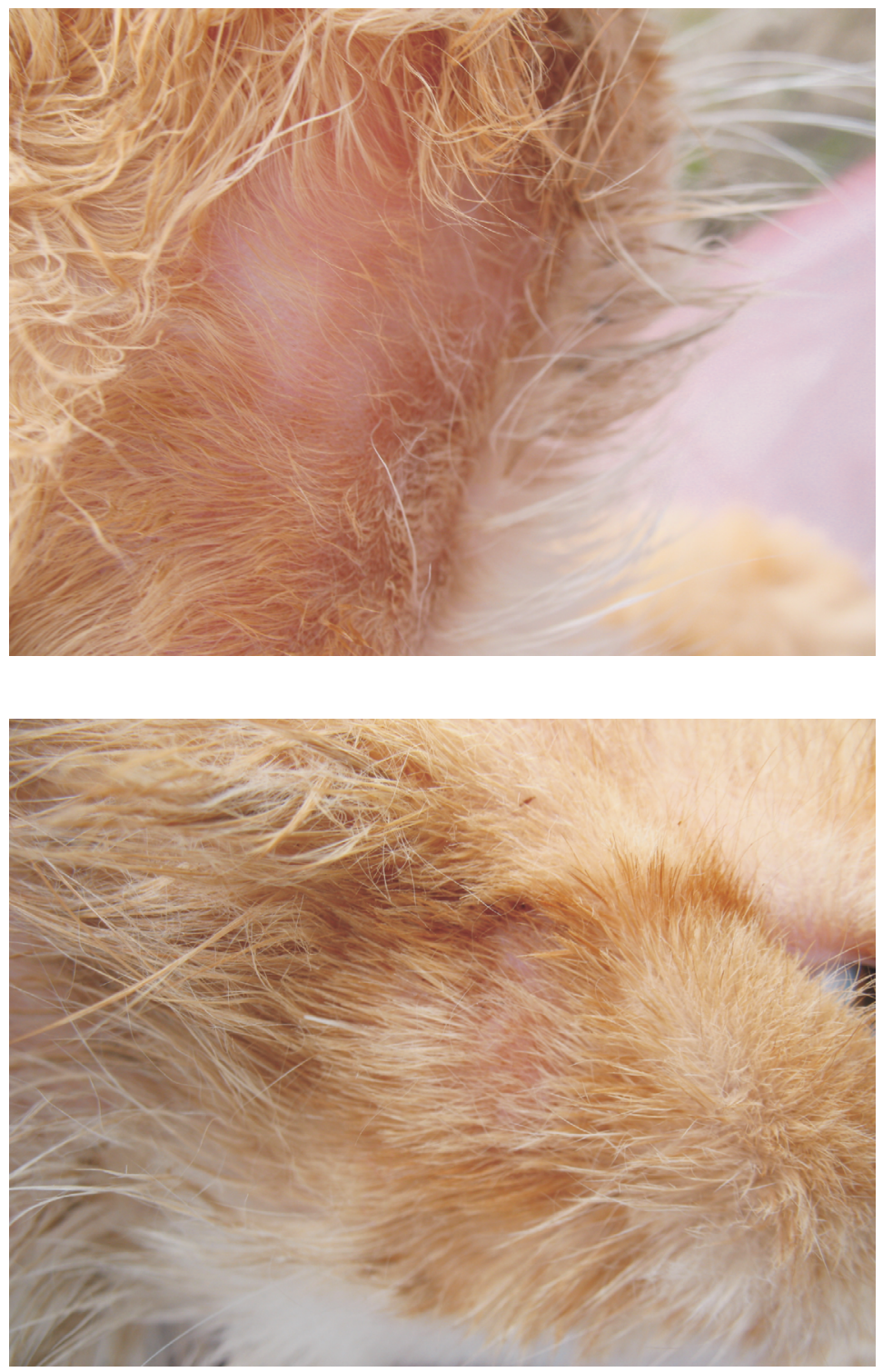

Figs. 6, 7. Lesions after 7 days of treatment with cream T-1.

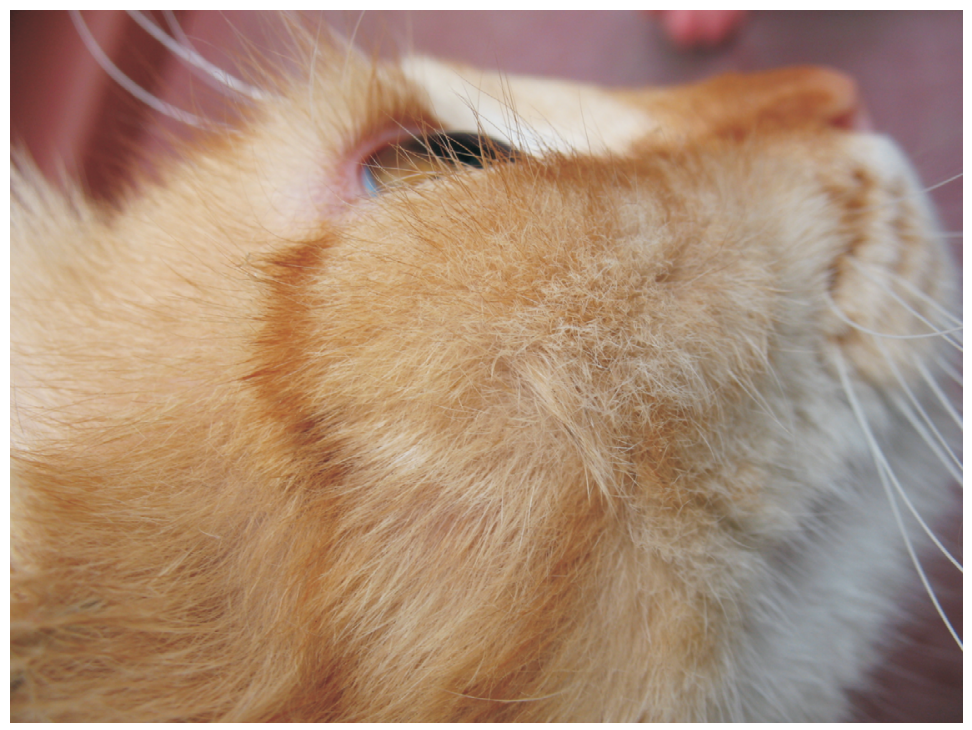

Fig. 8. Skin recovered after 15 days of treatment with cream T-1. 
cal treatment of the skin diseases. They are supplementary to classic treatments and help normalize barrier function of the stratum corneum; they suppress anti-inflammatory effects and make the epidermis more resistant to external stress factors. Salicylic acid is generally used in ointments and solutions because of its antiseptic, keratolytic and antipruritic properties; it increases hydration and softens the stratum corneum by decreasing its $\mathrm{pH}$. Topical salicylates improve the absorption and productiveness of other topical medications (Yosipovitch et al. 2001). Moreover, the $\mathrm{pH}$ of creams was adjusted to 6.2 , because reduction in skin $\mathrm{pH}$ suppresses the reproduction of pathogenic microbiota (Matousek et al. 2003).

The present results concur with those of previous studies, which demonstrated high effectiveness of topical terbinafine formulations in the treatment of experimental dermatophytosis in guinea pigs (Ghannoum et al. 2004, 2009, 2010).

Allylamines and azoles are lipophilic drugs; they usually accumulate in the stratum corneum and hair follicles, and persist there at concentrations above the MIC for several weeks after a short-term therapy (Jessup et al. 2000, Foust et al. 2007). Absorption of lipophilic drugs into the bloodstream is very low after topical application (Schafer-Korting et al. 2008). Presumably that was the reason the mycological test and toothbrush technique results were negative to all the cats at the end of the treatment after all clinical signs of infection disappeared.

This study demonstrated that terbinafine tended to have superior clinical efficacy compared to the azole tested. This apparent superiority may be due to the fungicidal activity and non-skin irritating properties of terbinafine compared to the fungistatic and irritating effect of the econazole. The inhibiting fungicidal activity of terbinafine, keratolytic and hydration effect of newly designed oil in water formulation allow reaching fast results of treating dermatophytosis in cats.

\section{References}

Ameen M (2010) Epidemiology of superficial fungal infections. Clin Dermatol 28: 197-201.

Bechert U, Christensen JM, Poppenga R, Fahmy SA, Redig $P$ (2010) Pharmacokinetics of terbinafine after single oral dose administration in red-tailed hawks (Buteo jamaicensis). J Avian Med Surg 24: 122-130.

Benson HA (2005) Transdermal drug delivery: penetration enhancement techniques. Curr Drug Deliv 2: 23-33.

Castanon-Olivares LR, Manzano-Gayosso P, Lopez-Martinez R, De la Rosa-Velazquez IA, Soto-Reyes-Solis E (2001) Effectiveness of terbinafine in the eradication of Microsporum canis from laboratory cats. Mycoses 44: 95-97.
Foust AL, Marsella R, Akucewich LH, Kunkle G, Stern A Moattari S, Szabo NJ (2007) Evaluation of persistence of terbinafine in the hair of normal cats after 14 days of daily therapy. Vet Dermatol 18: 246-251.

Frymus T, Gruffydd-Jones T, Pennisi MG, Addie D, Belhk S, Boucraut-Baralon C, Egberink H, Hartmann K, Hosie MJ, Lloret A, Lutz H, Marsilio F, Mostl K, Radford AD, Thiry E, Truyen U, Horzinek MC (2013) Dermatophytosis in cats: $\mathrm{ABCD}$ guidelines on prevention and management. J Feline Med Surg 15: 598-604.

Ghannoum MA, Hossain MA, Long L, Mohamed S, Reyes G, Mukherjee PK (2004) Evaluation of antifungal efficacy in an optimized animal model of Trichophyton mentagrophytes-dermatophytosis. J Chemother 16: 139-144.

Ghannoum MA, Long L, Kim HG, Cirino AJ, Miller AR, Mallefet P (2010) Efficacy of terbinafine compared to lanoconazole and luliconazole in the topical treatment of dermatophytosis in a guinea pig model. Med Mycol 48: 491-497.

Ghannoum MA, Long L, Pfister WR (2009) Determination of the efficacy of terbinafine hydrochloride nail solution in the topical treatment of dermatophytosis in a guinea pig model. Mycoses 52: 35-43.

Ivaskiene M, Matusevicius A, Grigonis A, Zamokas G, Siug zdaite J (2011) Establishing the efficacy of novel topical formulations in the treatment of experimental dermatophytosis in guinea pigs. Vet Med Zoot 54: 26-34.

Jessup CJ, Ryder NS, Ghannoum MA (2000) An evaluation of the in vitro activity of terbinafine. Med Mycol 38: 155-159.

Korting HC, Kiencke P, Nelles S, Rychlik R (2007) Comparable efficacy and safety of various topical formulations of terbinafine in tinea pedis irrespective of the treatment regimen: results of a meta-analysis. Am J Clin Dermatol 8: 357-364.

Kotnik T (2002) Drug efficacy of terbinafine hydrochloride (Lamisil) during oral treatment of cats, experimentally infected with Microsporum canis. J vet Med B Infect Dis and Vet Public Health 49: 120-122.

Kotnik T, Cerne M (2006) Clinical and histopathological evaluation of terbinafine treatment in cats experimentally infected with Microsporum canis. Acta Vet Brno 75: 541-547.

Maertens JA (2004) History of the development of azole derivatives. Clin Microbiol Infect 10 (Suppl 1): 1-10.

Matousek JL, Campbell KL, Kakoma I, Schaeffer DJ (2003) The effects of four acidifying sprays, vinegar, and water on canine cutaneous $\mathrm{pH}$ levels. J Am Anim Hosp Assoc 39: 29-33.

Matusevicius A, Ivaskiene M, Spakauskas V (2008a) Antifungal drugs. Part I. Fungal cell structure, function and susceptible targets for antifungal agents. Review. Vet Med Zoot 43: 3-13.

Matusevicius A, Ivaskiene M, Spakauskas V (2008b) Part II Antifungal drugs, antifungal substance, compounds and drugs. Review. Vet Med Zoot 44: 3-22.

Moriello KA (2004) Treatment of dermatophytosis in dogs and cats: review of published studies. Vet Dermatol 15: 99-107.

Ozcan I, Abaci O, Uztan AH, Aksu B, Boyacioglu H, Guneri T, Ozer O (2009) Enhanced topical delivery of terbinafine hydrochloride with chitosan hydrogels. AAPS PharmSciTech 10: 1024-1031.

Sakai MR, May ER, Imerman PM, Felz C, Day TA, Carlson SA, Noxon JO (2011) Terbinafine pharmacokinetics 
after single dose oral administration in the dog. Vet Dermatol 22: 528-534.

Schafer-Korting M, Schoellmann C, Korting HC (2008) Fungicidal activity plus reservoir effect allow short treatment courses with terbinafine in tinea pedis. Skin Pharmacol Physiol 21: 203-210.

Scott DW, Miller WH, Griffin CE (2001) Fungal skin diseases. In: Muller and Kirk's Small Animal Dermatology. WB Saunders, Philadelphia, pp 336-361.

Seebacher C, Bouchara JP, Mignon B (2008) Updates on the Epidemiology of Dermatophyte Infections. Mycopathologia 166: 335-352.

Sheppard D, Lampiris HW (2007) Antifungal agents. In: Katzung BG (ed) Basic and clinical pharmacology, 10th ed., McGraw-Hill Companies, pp 787-788.

Skerlev M, Miklic P (2010) The changing face of Microsporum spp. infections. Clin Dermatol 28: 146-150.
Vanden Bossche H, Engelen M, Rochette F (2003) Antifungal agents of use in animal health-chemical, biochemica and pharmacological aspects. J Vet Pharmacol Ther 26: 5-29.

Wang A, Ding H, Liu Y, Gao Y, Zeng Z (2012) Single dose pharmacokinetics of terbinafine in cats. $\mathrm{J}$ Feline Med Surg 14: 540-544.

Williams A (2003) Transdermal and topical drug delivery: From theory to clinical practice. 1st ed., Pharmaceutical Press, London.

Williams MM, Davis EG, KuKanich B (2011) Pharmacokinetics of oral terbinafine in horses and Greyhound dogs. J Vet Pharmacol Ther 34: 232-237.

Yosipovitch G, Sugeng MW, Chan YH, Goon A, Ngim S, Goh CL (2001) The effect of topically applied aspirin on localized circumscribed neurodermatitis. J Am Acad Dermatol 45: 910-913. 